\title{
Segmentation of Vector Fields by Critical Point Analysis: Application to Brain Deformation
}

\author{
Gert Wollny, Marc Tittgemeyer, and Frithjof Kruggel \\ Max-Planck-Institute of Cognitive Neuroscience, Stephanstr. 1a, 04277 Leipzig, Germany \\ wollny@cns.mpg.de, tittge@cns.mpg.de, kruggel@cns.mpg.de
}

\begin{abstract}
MRI examinations may be used to monitor the progress of neurological disease. Arising structural changes can then be quantified using non-rigid registration procedures. However, the interpretation of the resulting large scale vector fields is difficult without further processing. We propose using contraction mapping to detect critical points such as attractors and repellors in order to characterize deforming areas. With the application to time series images we show, that critical points help to get a better perception of the brain deformation and the underlying pathological process.
\end{abstract}

\section{Introduction}

The progress of neurological diseases, (e.g. neoplasm growth, scarification, and atrophies) may be monitored by a time series of MRI-examinations. MR images are obtained as $3 \mathrm{D}$ matrices of intensity values. Though visual comparison of the image data is still the gold standard in clinical neuro-science, with the development of modern image processing tools, automated image analysis gains ground. An automated analysis of time series image data is usually preceded by rigid registration [13], to align brains with respect to position and orientation. Then, the application of nonrigid registration [2] yields a large scale vector field mapping one image onto another, and thereby, reflecting structural changes. Methods have been presented, to segment areas of changing brain volume by using the Jacobian [3,11], or to detect growth pattern by using tensor maps [14].

We propose the usage of critical points, as they where introduced by Abraham et al. [1], to improve perception of the vector field and consequently, to improve the understanding of the monitored disease process. Classifying these points by their so called phase portraits will help to localize interesting spots, such as, growth centers and centers of matter loss, and it will help to interpret the observed pathological process.
However, the detection and visualization of critical points is still an active research area where rather sophisticated mathematical methods have been employed [5]. Established are topological methods that decompose vector fields in different global regions of interest based on local linear [6] or higher-order [12] approximations of the Jacobian. Philippou et al. [8] introduced a geometrical method where critical points are found at the intersection of lines tangent with the vector orientation (or at the intersection of planes orthogonal to the vectors). Methods based on the Poincaré-Hopf index theorem e.g., [4] are also widely employed.

Due to the finite spatial resolution of the images, the displacement field is given on a discrete grid. Since, for example, growth or atrophying processes take place in finite sub-compartments of the brain, representing critical points by point sources is an over-simplification. Most conventional methods therefore fail to detect critical points when applied to medical vector fields. Thus, in our application critical points are not regarded as infinitesimally small.

We rather propose a novel method to detect critical points that is based on the contraction mapping theorem [7]. For an application to a patient suffering from Alzheimer Disease we will illustrate how critical points analysis may help to understand the disease process.

\section{The Concept of Critical Points}

Consider a vector field $\vec{u}: \Omega \rightarrow \mathbb{R}^{3}$ for some compact domain $\Omega \subseteq \mathbb{R}^{3}$ and the set:

$$
U_{\varepsilon}\left(\vec{x}^{\prime}\right):=\left\{\vec{x} \mid\left\|\vec{x}-\vec{x}^{\prime}\right\|<\varepsilon, \vec{x} \in \Omega\right\},
$$

for any $\varepsilon>0, \varepsilon \in \mathbb{R}$ and a $\vec{x}^{\prime} \in \Omega$; the set $U_{\varepsilon}$ is called the $\varepsilon$-environment of $\vec{x}^{\prime}$.

The Taylor series expansion of $\vec{u}(\vec{x})$ about the point $\vec{x}^{\prime}$ yields:

$$
\vec{u}(\vec{x})=\left.\frac{\partial u_{i}}{\partial x_{j}}\right|_{\vec{x}^{\prime}}\left(\vec{x}-\vec{x}^{\prime}\right)+\vec{u}\left(\vec{x}^{\prime}\right)+o(\vec{x}) .
$$


By taking into account only its linear terms, and with the substitution $\mathbf{A}:=\left.\frac{\partial u_{i}}{\partial x_{j}}\right|_{\vec{x}^{\prime}}, \mathbf{A} \in \mathbb{R}^{3 \times 3}$ we obtain

$$
\vec{u}(\vec{x})=\mathbf{A}\left(\vec{x}-\vec{x}^{\prime}\right)+\vec{u}\left(\vec{x}^{\prime}\right) .
$$

Thus, we can now define cf. [8]:

A critical point $\vec{x}_{\mathrm{cp}}$ is an equilibrium point in the vector field topology where $\vec{u}\left(\vec{x}_{\mathrm{cp}}\right)=0$ while there exists an $\varepsilon>$ $0, \varepsilon \in \mathbb{R}$ so, that $\vec{u}(\vec{x}) \neq 0 \forall \vec{x} \in U_{\epsilon}\left(\vec{x}_{\mathrm{cp}}\right) \backslash\left\{\vec{x}_{\mathrm{cp}}\right\}$. Within the vicinity of a critical point $\vec{x}_{\mathrm{cp}}$, the vector field $\vec{u}(\vec{x})$ - as it is outlined in (2) - can be approximated by

$$
\vec{u}(\vec{x})=\mathbf{A}\left(\vec{x}-\vec{x}_{\mathrm{cp}}\right),
$$

where the matrix $\mathbf{A}$ is called the phase portrait of the critical point $\vec{x}_{\mathrm{cp}}$. As a first-order Taylor series would have a limited scope in modeling $\vec{u}(\vec{x})$ adequately, i.e., the influence of critical point $\vec{x}_{\mathrm{cp}}$ would decay with distance $\Delta \vec{x}=$ $\vec{x}-\vec{x}_{\mathrm{cp}}$, accuracy in modeling can be increased by introducing the attenuation factor $1 /\left\|\vec{x}-\vec{x}_{\mathrm{cp}}\right\|^{2}$. Consequently, the approximation of $\vec{u}(\vec{x})$ now reads

$$
\vec{u}(\vec{x})=\frac{1}{\left\|\vec{x}-\vec{x}_{\mathrm{cp}}\right\|^{2}} \mathbf{A}\left(\vec{x}-\vec{x}_{\mathrm{cp}}\right) .
$$

A critical point may be classified with respect to the eigenvalues of $\mathbf{A}$ (as proposed by Abraham et al. [1]): we distinguish attractors, repellors, saddle points, and rotation centers (see Fig. 1). For our intended application, namely to interpret morphological changes of the brain, attractors and repellors may be interpreted as areas of matter loss and growth, respectively, saddle points may correspond to configurations at barriers or membranes, and rotation centers may indicate local tissue shearing.

\subsection{Attractors and Repellors as Contraction Transformation Fix Points}

Given a complete metric space $(\Theta, d)$ with the distance function $d$ defined over the set $\Theta$ (cf. [7]). A mapping $f: \Theta \rightarrow \Theta$ from the metric space into itself is called a transformation. A transformation is called contractive on $\Theta$ if there is a constant $s \in[0,1[$ such that

$$
d(f(x), f(y)) \leq s \cdot d(x, y) \forall x, y \in \Theta .
$$

Any such number $s$ is called a contractivity factor for the transformation $f$.

Theorem 1 Let $f: \Theta \rightarrow \Theta$ be a contractive transformation on a complete metric space $(\Theta, d)$. Then the transformation $\mathrm{f}$ possesses exactly one point $a \in \Theta$ with $f(a)=$ $a$, and a is called fix point of transformation $f$. Moreover, for any $x \in \Theta$, the sequence $x, f(x), \ldots, f^{k}(x):=$ $f\left(f^{k-1}(x)\right), \ldots$ or short

$$
\left[f^{k}(x)\right]_{k=0,1, \ldots}
$$

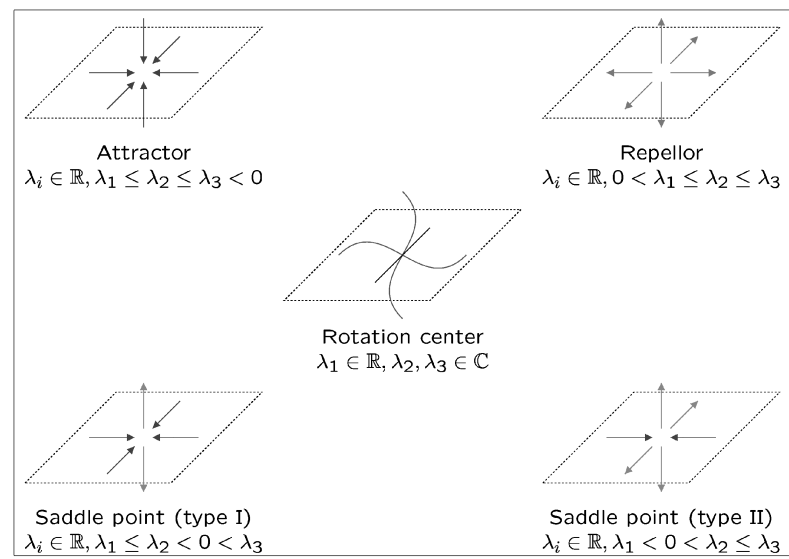

Figure 1. Classification criteria for critical points (after Abraham et al. [1]). $\lambda_{i}$ denote the eigenvalues of the phase portrait to a critical point.

converges to the fix point a, i.e.

$$
\lim _{k \rightarrow \infty} f^{k}(x)=a .
$$

For a proof of this theorem see, e.g., [7]. Fix point $a$ is also called the attracting point of set $\Theta$.

Consider now a vector field $\vec{u}(\vec{x})$ defined on the domain $\Omega$. Additionally we assume

$$
\vec{x}+\vec{u}(\vec{x}) \in \Omega \forall \vec{x} \in \Omega,
$$

which holds for vector fields obtained by non-rigid registration of images defined on $\Omega$.

With the Euclidean distance

$$
d(\vec{x}, \vec{y}):=\|\vec{x}-\vec{y}\|_{2} \forall \vec{x}, \vec{y} \in \Omega,
$$

the pair $(\Omega, d)$ defines a metric space.

Using an Euclidean reference frame and taking assumption (7) into account $\vec{u}(\vec{x})$ defines a transformation $T: \Omega \rightarrow$ $\Omega$ through

$$
T(\vec{x}):=\vec{x}+\vec{u}(\vec{x}) .
$$

For the limit of each Cauchy sequence $X=\left[\vec{x}_{k}\right]_{k=0,1, \ldots}$

$$
\lim _{k \rightarrow \infty} \vec{x}_{k}=\vec{x} \in \Omega,
$$

is in $\Omega,(\Omega, d)$ is a complete metric space [7].

Consider now a fix point $\vec{a} \in \Omega$. The set

$$
\Theta(\vec{a}):=\left\{\vec{x} \mid \vec{x} \in \Omega \wedge \lim _{k \rightarrow \infty} T^{k}(\vec{x})=\vec{a}\right\}
$$

is called the attraction area $\Theta$ of $\vec{a}$. For a consideration of the phase portrait $\mathbf{A}_{\vec{a}}$ and especially its eigenvalues we will 
now take only fix points into account that have the following property: It exists a positive number $\delta>0$ with

$$
\|\vec{u}(\vec{x})\| \neq 0 \forall \vec{x} \in U_{\delta}(\vec{a}) \backslash\{\vec{a}\} \wedge U_{\delta}(\vec{a}) \subseteq \Theta
$$

i.e. the attracting area of fix point $\vec{a}$ consist of at least a sphere with radius $\delta$ around fix point $\vec{a}$, and the vector field is not zero in this sphere.

For the estimation of the eigenvalues we consider a subset of $\Theta$

$$
\tilde{\Theta}:=\{\vec{x} \mid \vec{x} \in \Theta \wedge\|\vec{x}-\vec{a}\|<\varepsilon, \varepsilon \geq \delta\} .
$$

Using (3) yields

$$
\mathbf{A}(\vec{x}-\vec{a}) \approx\|\vec{x}-\vec{a}\|^{2} \vec{u}(\vec{x}) .
$$

Since $\vec{a} \rightarrow \vec{x}+\vec{u}(\vec{x})$ for $\vec{x} \rightarrow \vec{a}$ and with substitution $\lambda=$ $\|\vec{x}-\vec{a}\|^{2}$ we obtain

$$
\mathbf{A} \vec{u}(\vec{x}) \approx-\lambda \vec{u}(\vec{x}) .
$$

This accounts for all vectors $\vec{u}(\vec{x}) \in \tilde{\Theta}$ and due to (12) $\tilde{\Theta}$ also contains a set of eigenvectors $\vec{u}_{i} \in \tilde{\Theta}$ of $\mathbf{A}_{\vec{a}}$. Thus all its three eigenvalues are less then zero $\lambda_{1} \leq \lambda_{2} \leq \lambda_{3}<$ 0 and fix point $\vec{a}$ is an attractor as can be seen from the classification in figure 1 .

By replacing transformation (9) with

$$
T_{\mathrm{inv}}(\vec{x}):=\vec{x}-\vec{u}(\vec{x})
$$

the fix points of sequences $\left[T_{\text {inv }}^{k}(\vec{x})\right]_{k=0,1, \ldots}$ yield repellors. The derivation is similar to the one given above.

\section{Estimation algorithm}

We will now describe the procedure to estimate attractors when using transformation $T$ (9), repellors when using $T_{\text {inv }}$ (16) respectively. We introduce a regular grid $\hat{\Omega}$ by discretizing the domain $\Omega$ based on the resolution of the studied input images.

\subsection{Cumulation}

To find fix points $\vec{a}$ in the deformation field $\vec{u}$ we first define a counter $C$ on $\hat{\Omega}$. Defining a threshold $t$, and

$$
\hat{\Omega}_{\mathrm{start}}:=\{\vec{x} \mid\|\vec{u}(\vec{x})\|>t, t>0, \vec{x} \in \hat{\Omega}\}
$$

yields a set of sequence start points, that ensures attraction areas $\Theta(\vec{a}) \supset\{\vec{a}\}$, i.e. the attraction area will contain more then just the starting point. Now we consider sequences $\left[T^{k}(\vec{x})\right]_{k=0,1, \ldots}$. When reaching the breaking condition $\vec{u}\left(T^{k}(\vec{x})\right)<t$ we increment counter $C\left(T^{k}(\vec{x})\right)$. After iteration over all $\vec{x} \in \hat{\Omega}_{\text {start }}$ the distribution of counter values $C(\vec{x})$ reflects the distribution of fix points of vector field $\vec{u}$.

\subsection{Clustering}

Since we work on a finite domain $\hat{\Omega}$ we will consider two sequences $\left[T^{k}\left(\vec{x}_{1}\right)\right]_{k=0,1, \ldots}$ and $\left[T^{l}\left(\vec{x}_{2}\right)\right]_{l=0,1, \ldots}$ to converge to the same fix point $\vec{a}$ if for a certain value $\alpha>0$, $\alpha \in \mathbb{R}$ if the following assumption is fulfilled:

$$
\begin{aligned}
& \vec{u}\left(T^{k}\left(\vec{x}_{1}\right)\right)<t \wedge \vec{u}\left(T^{l}\left(\vec{x}_{2}\right)\right)<t \rightarrow \\
& \quad\left\|T^{k}\left(\vec{x}_{1}\right)-T^{l}\left(\vec{x}_{2}\right)\right\|<\alpha .
\end{aligned}
$$

Here $\alpha$ should be chosen based on the resolution of the grid $\hat{\Omega}$; a convenient value is half a pixel size.

Attracting points are finally obtained by clustering counter $C$ : First, find a seed point $\vec{s} \in \hat{\Omega}$ with $C(\vec{s})>t$, and create a point size cumulation area $\Theta_{\vec{s}}$. Then, the cumulation area $\Theta_{\vec{s}}$ is grown by adding neighboring grid points $\vec{x}$ as long as $C(\vec{x})>t$. After the growth of area $\Theta_{\vec{s}}$ is finished, its center of gravity is calculated, weighting with the counter values $C(\vec{x}), \vec{x} \in \Theta_{\vec{s}}$ and then used as critical point location:

$$
\vec{x}_{\mathrm{cp}}:=\frac{\sum_{\vec{x} \in \Theta_{\vec{s}}} C(\vec{x}) \vec{x}}{\sum_{\vec{x} \in \Theta_{\vec{s}}} C(\vec{x})} .
$$

Finally, for each $\vec{x} \in \Theta_{\vec{s}}$ set $C(\vec{x}):=0$.

Repeat this procedure until $C(\vec{x}) \leq t \forall \vec{x} \in \hat{\Omega}$.

\subsection{Phase Portrait Estimation and Classification}

Using the approximation of vector field $\vec{u}(\vec{x})$ in the environment of a critical point $\vec{x}_{\mathrm{cp}}(3)$, and substituting $\Delta \vec{x}:=$ $\vec{x}-\vec{x}_{\mathrm{cp}}$ yields

$$
\|\Delta \vec{x}\| \vec{u}\left(\vec{x}_{\mathrm{cp}}+\Delta \vec{x}\right)=\mathbf{A}(\Delta \vec{x}) .
$$

Considering a certain environment of $\vec{x}_{\mathrm{cp}}$ and taking (20) into account, we obtain an over-determined system of linear equations [8] that can be solved using Householder transformations [10]. Since $\mathbf{A}$ is a $3 \times 3$ matrix, calculating the eigenvalues and thus a classification of critical point $\vec{x}_{\mathrm{cp}}$ can easily be done solving

$$
\operatorname{det}(\mathbf{A}-\lambda \mathbf{I})=0
$$

using Cardan's formula [10].

\section{Application}

A patient suffering from Alzheimer Disease (AD) was scanned twice in an 12 month interval. After rigid registration [13], both datasets were registered using the fluid dynamic, non-rigid approach described by Christensen [2]. We obtained a displacement vector at each point of the reference image, corresponding to the shift of tissue during the 
time interval. From the displacement field we extracted critical points. To represent their properties, a color scheme is implemented, where green and red indicate repelling or attracting property, and blue a rotation component. With this color-code a critical point could be easily visualized: a repellor appears purely green, an attractor red, and a rotation center blue. Different types of saddle points may be distinguished by mixing the respective colors.

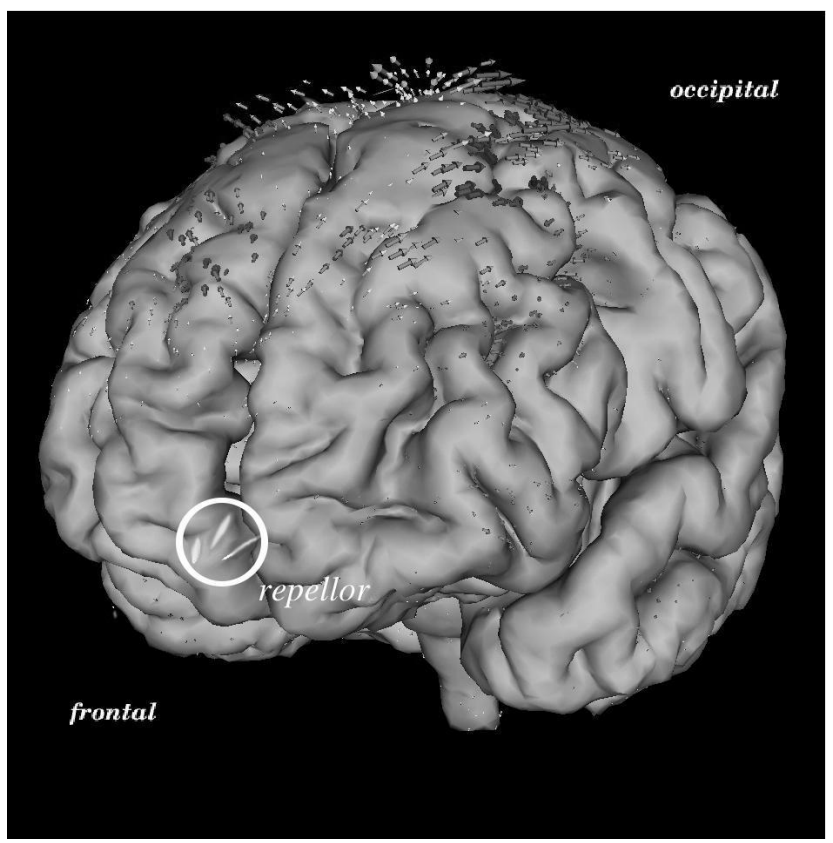

Figure 2. Pattern of shape change of a patients' brain between two examination time points. The critical point (repellor) within the frontal CSF compartment indicates a virtual flow in fronto-occipital direction. The major displacement lines (arrows) depict the deformation lines.

The set of critical points is dominated by a strong repellor located in the pre-frontal cerebro-spinal fluid (CSF) compartment (Fig. 2). The bifrontal tissue loss induced by the disease leads to an increase of the frontal CSF compartment. Displacement stream lines (Figs. 2 map the "flow" of tissue along the mid line structures (as a correlate of a global atrophy) and reveal a retraction of the brain in the frontal-occipital direction. As could be deduced by Fig. 2 the strongest deformations occur in the posterior portions of the first and second frontal gyrus on both hemispheres.

\section{Conclusion}

We proposed to describe deformation fields obtained from non-rigid registration of time series of MR images by its critical points. A novel method for finding critical points in discrete vector fields was introduced based on contraction mapping.

However, our method fails to detect rotation centers or balanced saddle points. Here, local measures based on the Jacobian (Helman et al. [6]) or global approaches like recently introduced by Polthier et al. [9] may promise better results.

\section{References}

[1] R. Abraham and C. Shaw. Dynamics: The Geometry of Behavior. Addiso-Wesley, Reading MA, 1992.

[2] G. E. Christensen. Deformable shape models for neuroanatomy. DSc.-thesis, Server Institue of Technology, Washington University, Saint Louis, 1994.

[3] M. Chung, K. Worsley, T. Paus, C. Cherif, D. Collins, J. Giedd, J. Rapoport, and A. Evans. A Unified Statistical Approach to Deformation-Based Morphometry. NeuroImage, 2001:595-606, 2001. FK.

[4] D. H. Gottlieb and G. Samaranayake. The index of discontinuous vector fields. New York J. Math., 1:130-148, 1995.

[5] H. C. Hege and K. Polthier, editors. Mathematical visualization : Algorithms, applications and numerics. Springer, Berlin, 1998.

[6] J. L. Helman and L. Hesselink. Representation and display of vector field topology in fluid flow data sets. IEEE Computer, 22(8):27-36, August 1989.

[7] N. Lu. Fractal Imaging. Academic Press, San Diego, CA, 1997.

[8] P. A. Philippou and R. N. Strickland. Vector field analysis and synthesis using three-dimensional phase portraits. Graphical Models And Image Processing, 6(59):466-462, 1997.

[9] K. Polthier and E. Preuß. Detecting vector field singularities from potentials. http://www-sfb288. math.tu-berlin.de/ kon$\mathrm{rad} /$ articles.html, 2001.

[10] W. H. Press, S. A. Teukolsky, W. T. Vetterling, and B. P. Flannery. Numerical Recipes in C. Cambridge University Press, New York, secon edition edition, 1992.

[11] D. Rey, G. Subsol, H. Delingette, and N. Ayache. Automatics detection and segmentation of evolving processes in $3 \mathrm{D}$ medical images: application to multiple sclerosis. In K. A. Šámal M. Todd-Pokropek A, editor, Information Processing in medical imaging, volume 1613 of Lect. Notes Comp. Sci, pages 154-167. Springer-Verlag, Berlin · Heidelberg, 1999.

[12] G. Scheuermann, H. Hagen, and H. Krüger. Clifford algebra in vector field visualization. In H.-C. Hege and K. Polthier, editors, Mathematical Visualization, pages 343-351. Springer-Verlag, Heidelberg, 1998.

[13] P. Thévenaz, U. E. Ruttimann, and U. M. Iterative multiscale registration without landmarks. In Proc. IEEE Int. Conf. on Image Processing, volume III, pages 228-231, Washington, DC, USA, October 23-26 1995. IEEE.

[14] P. M. Thompson, J. N. Giedd, R. P. Woods, D. MacDonals, A. C. Evans, and A. W. Toga. Growth patterns in the developing human brain detected using continuum-mechanical tensor mapping. Nature, 404(6774):190-193, March 2000. 